\title{
High-Energy Cosmic Rays
}

\author{
Barbara De Lotto*† \\ University of Udine and INFN Trieste, Italy \\ E-mail: barbara.delotto@uniud.it
}

In the past few years significant progress has been made towards identifying and understanding cosmic particle accelerators. Nevertheless some critical, long-standing questions remain unanswered, and the field is as active as ever.

I review recent experimental results on high-energy (HE, $>10^{15} \mathrm{eV}$ ) cosmic rays (CR). I will focus on direct $\mathrm{CR}$ electron and positron measurements and indirect ultra-HE $\left(>10^{18} \mathrm{eV}\right) \mathrm{CR}$ measurements, and on Very-HE ( $>100 \mathrm{GeV}$ ) gamma ray detections.

Open questions and prospects are addressed, and proposed and upcoming experiments are outlined.

European Physical Society Conference on High Energy Physics - EPS-HEP2019 -

10-17 July, 2019

Ghent, Belgium

\footnotetext{
* Speaker.

${ }^{\dagger}$ I thank Alessandro De Angelis and Massimo Persic for carefully reading this paper.
} 


\section{Introduction}

We call cosmic rays (CR) the high-energy (above $\sim 1 \mathrm{GeV}$ ) particles of extraterrestrial origin incident at the terrestrial atmosphere. These particles are dominantly protons, with some $10 \%$ of helium nuclei, and a small, albeit very important, fraction of electrons, positrons, gamma rays and neutrinos. We observe cosmic rays particles reaching the Earth up to energies $\sim 10^{20} \mathrm{eV}$ from not yet understood extraterrestrial accelerators. Despite the enormous progress made by particle acceleration technology, the energies reached by ground accelerators are limited to some $\mathrm{TeV}$. High-energy particles from space are a powerful tool to investigate fundamental physics and interactions, and understanding the processes by which they are generated can make us access to new astrophysical phenomena. We are aware of the existence of charged particle radiation of cosmic origin since more then a century, but still we do not know what sources originate them nor what mechanisms are capable of conferring such energies.

Charged cosmic rays are deflected by extragalactic (order of $1 \mathrm{nG}-1 \mathrm{fG}$ ) or by galactic (order of $1 \mu \mathrm{G}$ ) magnetic fields. The curvature induced by astronomical magnetic fields

$$
\frac{R_{L}}{1 \mathrm{kpc}} \simeq \frac{E / 1 \mathrm{EeV}}{B / 1 \mu \mathrm{G}}
$$

is such that it is impossible to locate sources close to the Galactic Center, about $9 \mathrm{kpc}$ away from us, for protons less energetic than $\sim 10 \mathrm{EeV}\left(1 \mathrm{EeV}=10^{18} \mathrm{eV}\right)$. There is little room from this energy to the drastic suppression due to the interaction with the Cosmic Microwave Background photons (CMB), the so-called GZK effect [1], happening in particular through the processes

$$
\begin{aligned}
p+\gamma \rightarrow \Delta & \rightarrow p+\pi^{0} \\
& \rightarrow n+\pi^{+} .
\end{aligned}
$$

Gamma rays originating from several cosmic production sites, even though much less numerous than charged CR of the same energy, being neutral can travel long distances without deflection by magnetic fields. Hence they allow to directly localize and study the emission sources. Finally, evidence for astrophysical neutrinos and, most recently, gravitational waves has paved the way for a new field of research called multi-messenger Astrophysics.

$\mathrm{CR}$ hitting the atmosphere at high energies (photons above $100 \mathrm{GeV}$ and nuclei above some $1 \mathrm{TeV}$ ) produce secondary particles that can reach the Earth's surface through the phenomenon of multiplicative showers. Above $100 \mathrm{TeV}$ the rate of primary $\mathrm{CR}$ is $<1 \mathrm{~m}^{-2} \mathrm{yr}^{-1}$ and direct observation in the upper layers of the atmosphere (balloon or aircraft) or above (spacecraft) is inefficient. Indirect measurements are done by experiments which exploit the atmosphere as a giant calorimeter.

A global view of the charged CR energy spectrum is given in Figure 1 [2]. It covers twelve orders of magnitude in energy and is approximately described by a power law $E^{-p}$, with $p$ around 3 on average. More in detail, after a sub-GeV energy region dominated by $\mathrm{CR}$ produced or moderated by the Sun, the spectrum becomes steeper ( $p \sim 2.7$ ) for energies $\lesssim 1000 \mathrm{TeV}$, i.e. $1 \mathrm{PeV}$; we know that this energy region is dominated by CR produced by astrophysical sources in our Galaxy. For higher energies a further steepening occurs $(p \sim 3.3)$, the region at which this change of slope takes place being called the "knee". The transition between galactic and extragalactic CR takes 
place at energies above the knee. For even higher energies $\left(\gtrsim 10^{18} \mathrm{eV}\right.$, i.e., $\left.1 \mathrm{EeV}\right)$ the $\mathrm{CR}$ spectrum becomes less steep, resulting in another change of slope, called the "ankle". Finally, at the highest energies, a drastic suppression is present.

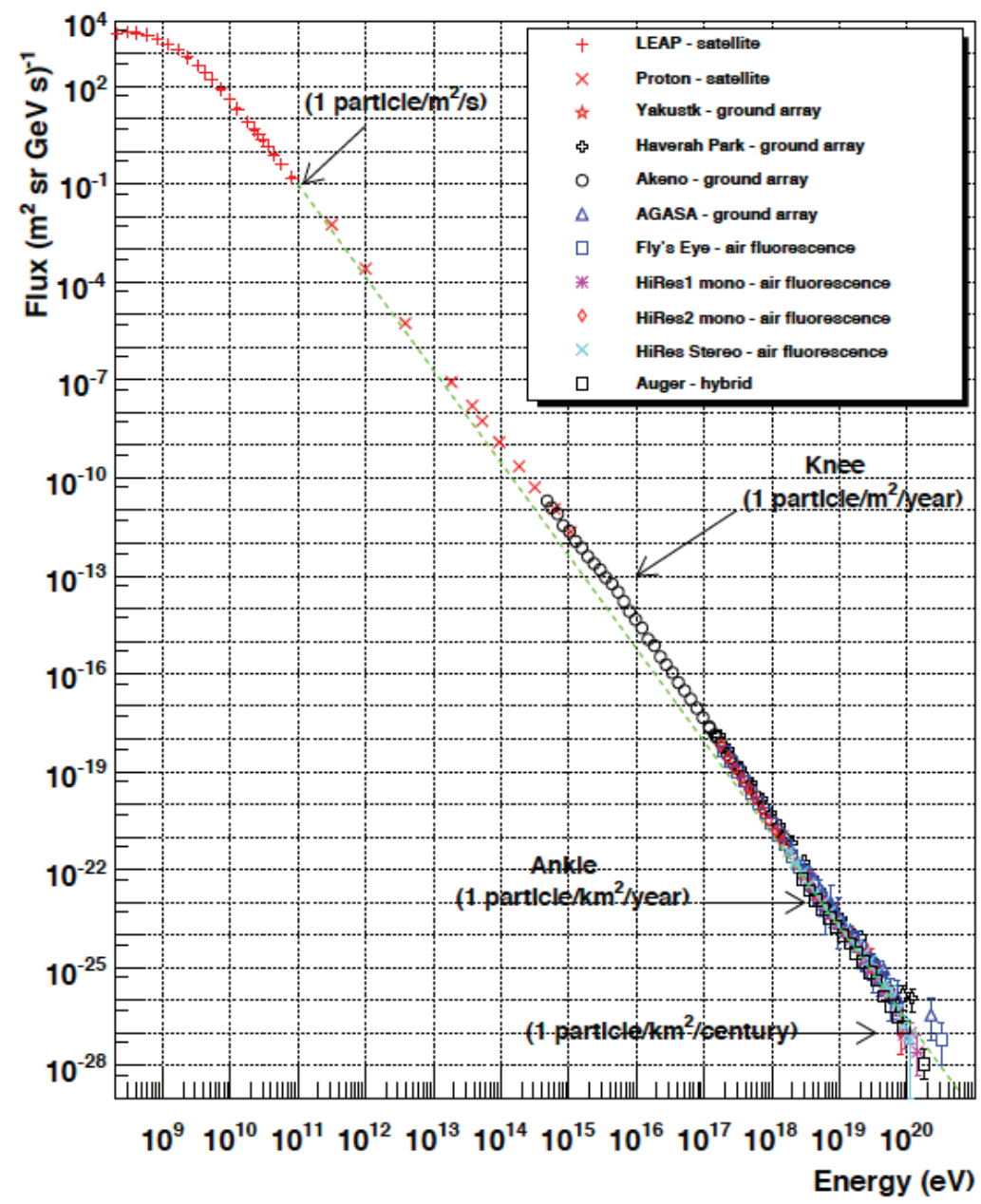

Figure 1: Energy spectrum of charged cosmic rays (from Ref. [2]).

There is a substantial consensus that galactic CR are somehow related to one or more types of supernova ( $\mathrm{SN}$ ) explosions and that acceleration is mainly due to diffusive transport in the proximity of strong shocks formed as a consequence of these explosions. Still a clear experimental evidence that $\mathrm{SN}$ can provide energies up to the knee is lacking. The transition zone between galactic and extragalactic CR must be above the knee, even though where is still a matter of debate. Possible acceleration sites are the accretion of supermassive (up to billion solar masses) black holes in the center of galaxies, the so called Active Galactic Nuclei (AGN): part of the gravitational energy released in the accretion of matter is transformed into the acceleration of protons or heavier nuclei.

As CR propagate to the Earth they lose energy scattering off the pervasive radiation fields and matter permeating the Universe. 
In this paper I will concentrate on recent direct measurements of charged cosmic electrons, positrons and protons, indirect measurements of Ultra High-Energy Cosmic Rays (UHECR, E > $10^{18} \mathrm{eV}$ ), and Very High-Energy (VHE, E > $100 \mathrm{GeV}$ ) gamma rays.

\section{Charged Cosmic Rays}

Several different kinds of detectors are necessary to study CR over the whole energy range. The strong decrease in CR flux with energy poses a big challenge to the size and the running times of the experimental installations when high energies are studied. Direct detection of CR at energies above a few hundred $\mathrm{GeV}$ suffers from the limited detection area of balloon and satelliteborn detectors. Above $1 \mathrm{PeV}$ observations are performed by exploiting the cascade induced in the atmosphere by the interactions of $\mathrm{CR}$ and ions.

\subsection{Direct measurements}

High-energy electrons and positrons account for only $\sim 1 \%$ of the total CR flux. Having short propagation distances (less than a few hundred parsec), their spectra are expected to be dominated by local electron accelerators or by the decay/interactions of heavier particles nearby. Positrons in particular could be the signature of the decay of dark matter particles.

The AMS-02 experiment on the International Space Station has recently published precision measurements on primary positrons [3], based on 1.9 million events corresponding to a factor of three increase in statistics compared to earlier published results, and electrons [4] based on 28.1 million events. The positrons and electron fluxes in the energy range $0.5 \mathrm{GeV}$ to, respectively, 1.0 and $1.4 \mathrm{TeV}$ are shown in Figure 2. The positron flux (Figure 2, left) shows a significant excess starting from $\sim 25 \mathrm{GeV}$, a sharp drop off above $\sim 285 \mathrm{GeV}$ and a finite energy cutoff of the source term around $E_{s}=810 \mathrm{GeV}$, established with a significance of more than $4 \sigma$, which could be interpreted as coming from astrophysical sources or from dark matter annihilation or decay. The flux is found to be consistent with isotropy. Contrary to the positron flux, the electron flux (Figure 2, right) does not show an energy cutoff below $1.9 \mathrm{TeV}$. In the entire energy range it is well described by the sum of two power law components. High-energy electrons could originate from different sources than high-energy positrons. Precision measurements of the $\mathrm{e}^{+}$and $\mathrm{e}^{-}$spectra in the $\mathrm{TeV}$ and multi- $\mathrm{TeV}$ energy range are crucial to clarify the problem [5].

Inclusive all electron $\left(\mathrm{e}^{+}+\mathrm{e}^{-}\right)$spectra have been reported by the CALET [6] and DAMPE[7] experiments. The current situation of all-electron spectrum direct measurements in space is presented in Figure 3 [8].

Measurements of the proton spectrum have also been reported. The DAMPE experiment has recently published the first direct measurement of cosmic ray protons up to $\sim 100 \mathrm{TeV}$ with high statistics [9], suggesting the existence of a new spectral feature of cosmic rays at energies lower than the knee, that could shed new light on acceleration and propagation mechanisms.

\subsection{Indirect measurements}

Indirect measurements through the detection of Extensive Air Showers (EAS) in the atmosphere are exploring the region above $0.5-1 \mathrm{PeV}$. Above $10^{18} \mathrm{eV}$ (UHECR), the two largest and 

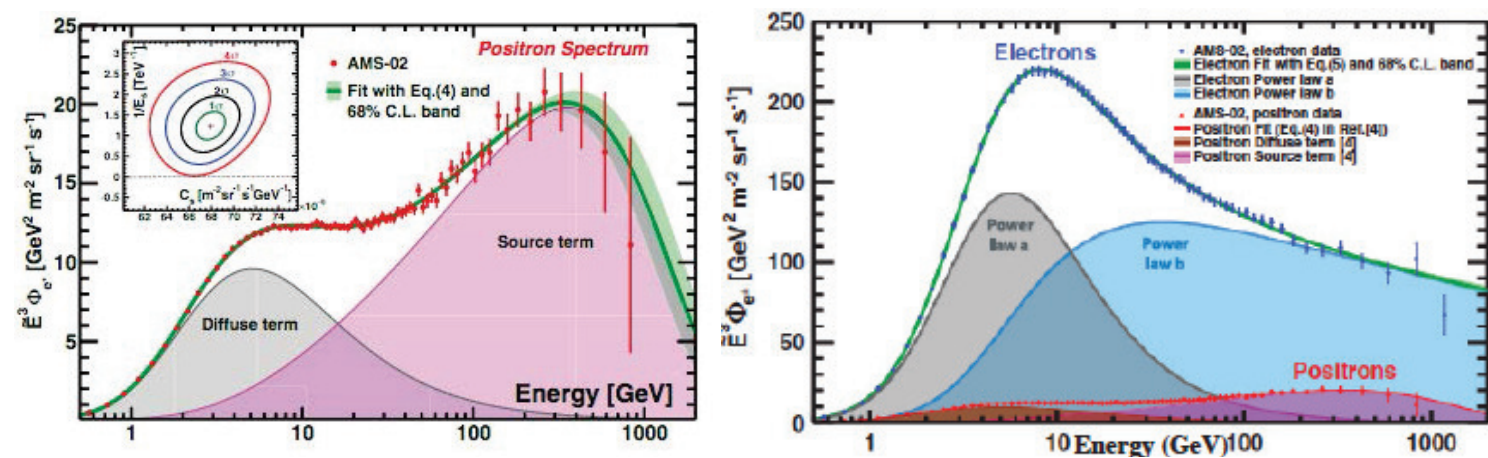

Figure 2: Left: Positron flux in the energy range [0.5-1000] GeV. The flux is parametrized as the sum of a diffuse term, describing the low-energy part dominated by the positrons produced in the collisions of ordinary cosmic rays with the interstellar gas, and a source term [3]. Right: Electron flux in the energy range [0.5-1400] GeV together with a fit described by the sum of two power law components [4].

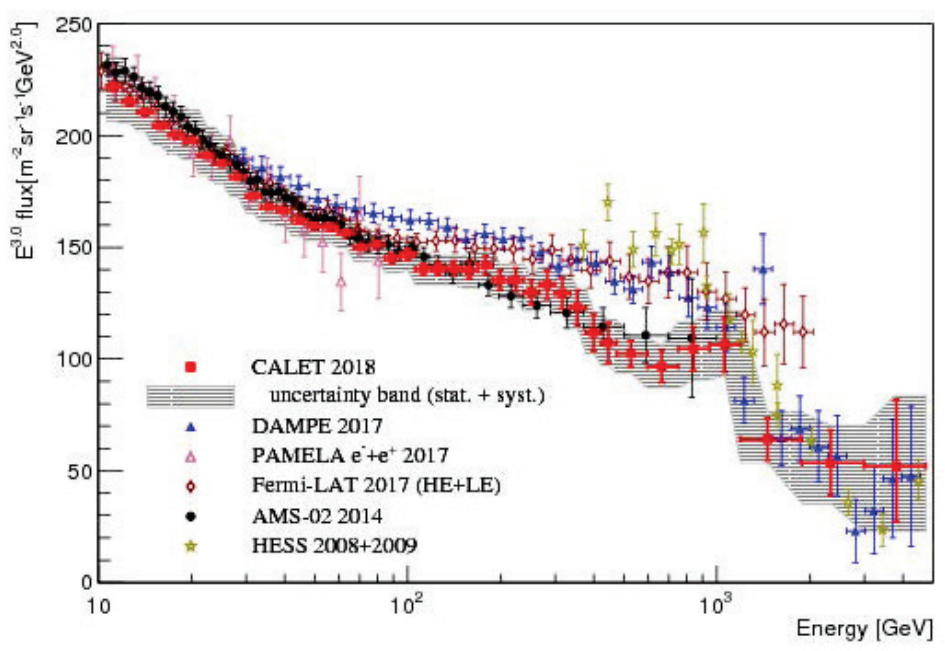

Figure 3: All-electron spectrum directly measured in space, together with the model calculation.

most precise detectors to date are the Pierre Auger Observatory [10] in Argentina and the Telescope Array [11] in Utah, USA. Both detectors exploit the hybrid concept, combining an array of surface detectors, to sample extensive air showers when they reach the ground, and telescopes overlooking the surface array, to collect the fluorescent light of the excited atmospheric nitrogen.

\subsubsection{Anisotropy}

The observed intensity of CR is characterised by a large degree of isotropy up to the highest energies. This fact indicates that turbulent magnetic fields inside the Milky Way are able to isotropize the Galactic part of the CR flux at least up to knee. The Pierre Auger Collaboration announced the observation of a large-scale anisotropy in the arrival directions of cosmic rays above $8 \times 10^{18} \mathrm{eV}$ [12]. This analysis was extended to cover more than three decades in energy, exploiting about 15 years of data. A total dipolar amplitude for $\mathrm{E}>8 \mathrm{EeV}$ is pointing $125^{\circ}$ away from the direction 
of the Galactic Center, as such indicating an extragalactic origin of the modulation. Hints of large scale anisotropy have also been reported by the Telescope Array experiment updating the hotspot analysis using the 10-years sample [13]. Figure 4 shows the CR flux in equatorial coordinates as measured by Auger.

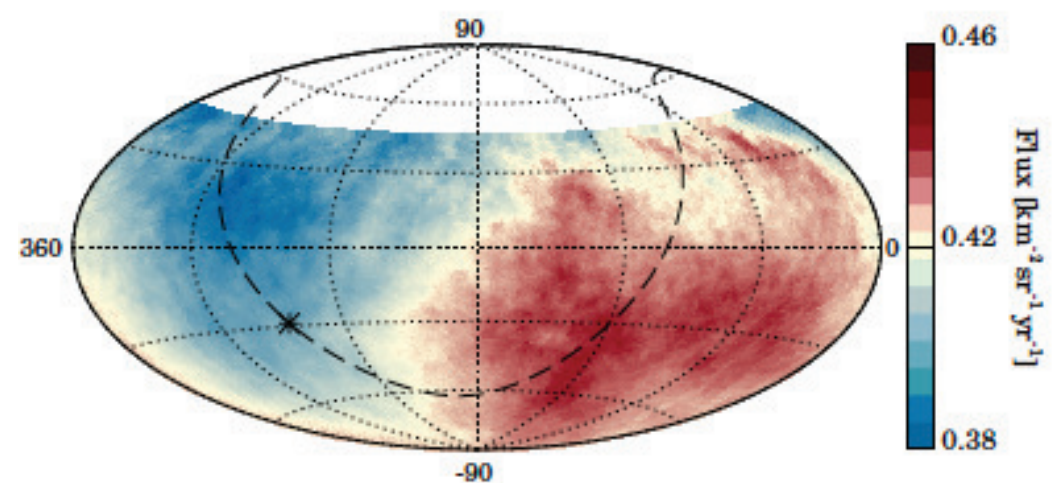

Figure 4: The skymap of the CR flux above $8 \mathrm{EeV}$ in equatorial coordinates. The Galactic plane and the Galactic center are indicated by a dashed line and a star respectively [12].

\subsubsection{Energy spectrum}

The high-energy CR spectra resulting from indirect measurements are shown in Figure 5 [14]. The flux is multiplied by $\mathrm{E}^{+2.6}$, highlighting a more complex structure with another change in the spectral index, the so-called "second knee", around $\sim 10^{17} \mathrm{eV}$, which could result from the overlap of spectra with different atomic number Z. Generally, the spectra are in agreement with each other when taking into account statistical, systematic, and scale uncertainties. Only at the very highest energies is there some tension between the flux measured by the Telescope Array and the Pierre Auger Observatory. Beside an ankle near $6 \times 10^{18} \mathrm{eV}$, a suppression of the UHECR flux above $\sim 5$ $\times 10^{19} \mathrm{eV}$ has been recently confirmed by Auger [15], obtained by combining a data set exploiting the total exposure of $\sim 80,000 \mathrm{~km}^{2} \mathrm{sr}$ yr. It may result from the destructive interaction with $\mathrm{CMB}$ photons, known as the GZK effect [1], or from an intrinsic limit to the maximum energies that can be attained in cosmic accelerators. A working group common to both the Auger and Telescope Array experiments has been set up to examine the differences in these features as well as in the overall energy scales. By focusing on the region of the sky commonly observed, the spectra are in substantial agreement within uncertainties when directional-exposure effects are taken into account [16].

\subsubsection{Mass composition}

The mass composition of UHECR is addressed using the measurements of the depth of maximum development of the shower in the atmosphere, $\mathrm{X}_{\max }$, and of its fluctuations. Auger and Telescope Array (TA) presented updates based on increased statistics and improvements in the analysis at the ICRC2019 Conference [17]. Auger clearly confirms a lighter composition around the ankle, which becomes heavier towards the second knee at lower energies as well as towards 


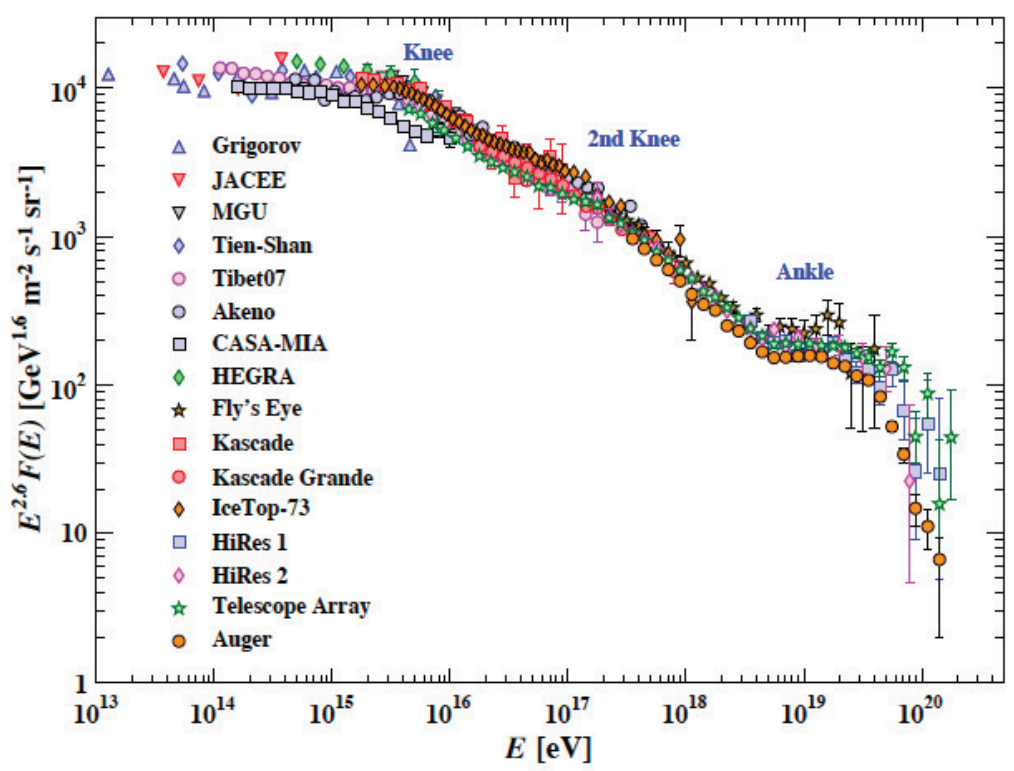

Figure 5: Energy spectrum of high-energy CR obtained from air-shower measurements [14].

the cut-off at higher energies (Figure 6, left). TA measurements (Figure 6, right) are compatible with a break in the elongation rate, but cannot exclude a constant value within present statistical uncertainties.
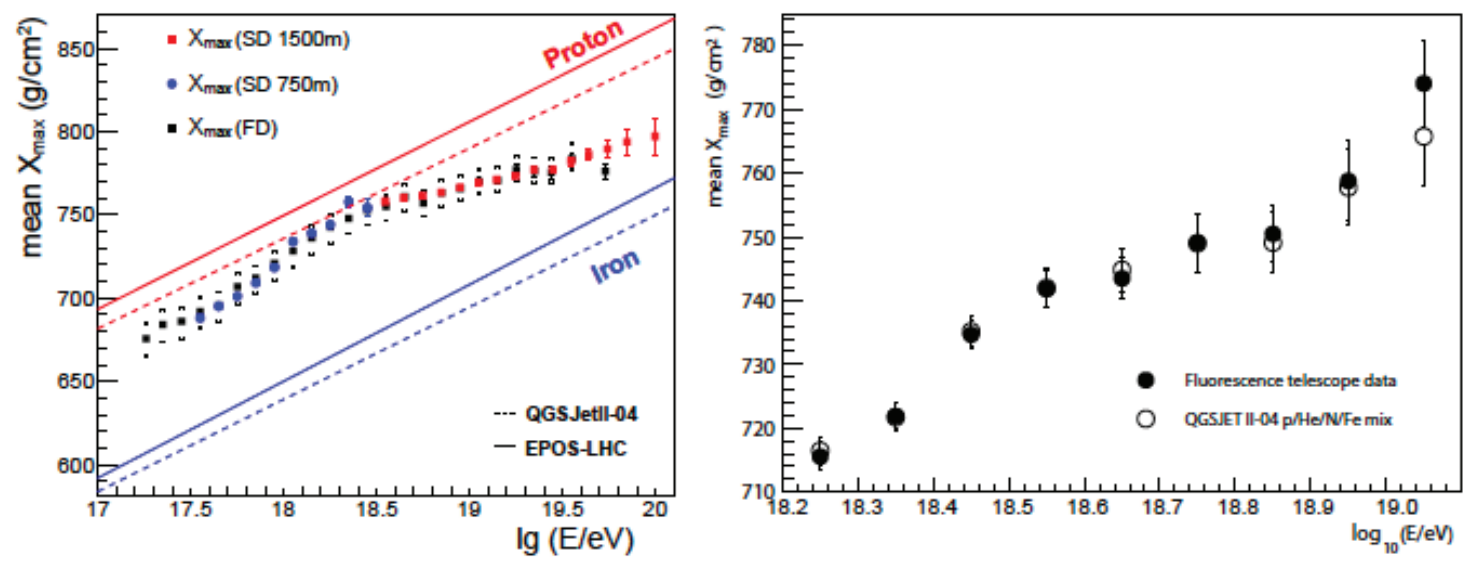

Figure 6: Mean $\mathrm{X}_{\max }$ measured by Auger (left) and by the Telescope Array (right) [17]

Hadronic interaction models remain one of the main systematic uncertainties in the interpretation of air-shower measurements. Understanding the hadronic and particle physics in air showers is an area of traditional cooperation with the particle physics community. The ongoing and planned measurements at accelerators, such as proton-oxygen collisions at LHC [18], will provide the basis for improvements in hadronic interaction models, finally resulting in a higher overall accuracy of indirect CR measurements. 


\section{Very High-Energy gamma rays}

Photons are historically the starry messengers for astronomy and astrophysics. Gamma rays constitute the most energetic end of the electromagnetic spectrum, and as such they are a unique tool to probe the most energetic processes in the Universe. Very High-Energy (VHE) gamma rays have, in the last few years, expanded our view of the Universe, probing the acceleration sites and propagation of CR. More than $220 \mathrm{VHE}$ gamma ray sources have now been established (http://tevcat2.uchicago.edu/). At these energies non-thermal mechanisms, where the photons produced are the result of nuclear transitions and of very-high-energy particle interactions, are probed.

\subsection{Instruments}

The rapid decrease of the gamma ray flux towards high energies requires large effective area detectors. Space-based detectors to directly measure the primary gamma rays are limited by the detector size, whose active area cannot realistically exceed $\sim 1 \mathrm{~m}^{2}$ because of the cost and difficulties of space technology. The Fermi-LAT [19], Agile [20], DAMPE [21] satellites are the main actors in this energy domain.

For energies above $\sim 30 \mathrm{GeV}$ electromagnetic air showers start to become detectable. A gamma ray hitting the atmosphere generates a cascade of secondary particles allowing indirect measurement methods of gamma rays. Extended surface arrays of detectors measure the secondary particles that reach the ground level. Such instruments have a high (close to 100\%) duty cycle and a large field of view (FoV). However their performance parameters, such as energy threshold and resolution as well as short term sensitivity, are rather poor. Currently only the Northern part of the sky is covered by surface arrays, HAWC [22] and Tibet AS+MD array [23].

Imaging Atmospheric Cherenkov Telescopes (IACT) measure the Cherenkov light produced by secondary charged particles in the air shower. They provide a complementary method for studying VHE gamma rays to surface arrays. The observations of IACTs are mostly limited to good weather and dark nights resulting in a low duty cycle $(\sim 11 \%)$. In addition the FoV of IACTs is usually only a few degrees across. However, their performance parameters, such as energy and angular resolution as well as short-term sensitivity and energy threshold allow in-depth studies of individual sources. The major IACTs currently operating are MAGIC [24] and VERITAS [25] in the Northern hemisphere, and H.E.S.S. [26] in the Southern one.

\subsection{Galactic physics}

One of the most important contributions of IACTs to Galactic TeV gamma-ray astronomy is the Galactic plane survey performed by H.E.S.S. with almost $2700 \mathrm{~h}$ of data [27]. The Galactic Plane scan allowed probing the population of various types of $\mathrm{TeV}$ sources in our Galaxy. Currently about 150 such sources are known. The observations of the Galactic center by H.E.S.S. resulted in a detection of a diffuse emission with a $1 / \mathrm{r}$ profile, which suggests a diffusion of CR injected in a quasi-continuous way from the central source [28]. This result has been recently confirmed by MAGIC using an alternative morphology analysis method [29].

Gamma rays above $100 \mathrm{TeV}$ are an excellent tracer to understand sources in the Galaxy that can accelerate hadrons up to $10^{15} \mathrm{eV}$ energy, the so called PeVatrons. Since gamma rays with such energies can also be produced in leptonic processes, it is important to measure the spectrum with 
sufficient accuracy that would allow discriminating between the leptonic and hadronic scenarios. The H.E.S.S. telescopes provided a very first evidence of a PeVatron in our Galaxy: the source is spatially located at the galactic center [28]. A natural candidate for looking for E $>100 \mathrm{TeV}$ emission is the Crab Nebula, the strongest stable source in the VHE gamma-ray sky. HAWC [30], Tibet AS+MD array [31] and MAGIC [32] reported detection of energies of $100 \mathrm{TeV}$ without any obvious cutoff (Figure 6).
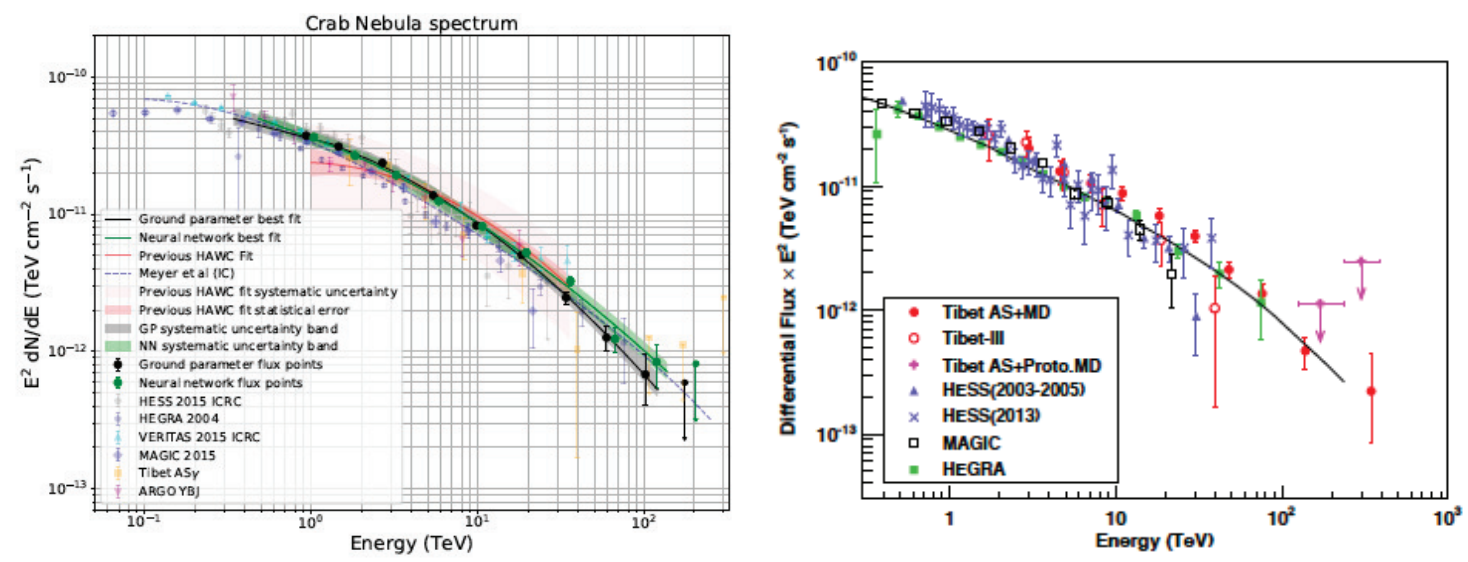

Figure 7: The Crab spectrum observed with: HAWC (left ) and Tibet AS+MD array (right)

\subsection{Extragalactic physics}

The recent detection of gamma ray emission from the TXS 0506+056 blazar with the Fermi LAT and MAGIC telescopes consistent with the arrival direction of the high-energy neutrino IceCube event 170922A [33] revived interest in hadronic models of gamma-ray emission. This event opened the era of multi-messenger astronomy with neutrinos [34].

\section{Future instruments}

Several upgrades of the existing instruments and new projects are on going and will increase the measurement accuracy over a wide range of energies.

AugerPrime, the ongoing upgrade of the Pierre Auger Observatory, includes various improvements of the $3000 \mathrm{~km}^{2}$ surface detector, in particular, the addition of a scintillation detector and a radio antenna to each water-Cherenkov tank, together with underground muon detectors in a denser part of the surface array [15]. Telescope Array will quadruple the size of the surface array of scintillation detectors to almost comparable size of Auger [35].

A new surface array in the Southern hemisphere is also considered (SWGO, [36]).

In China the LHAASO multi-detector experiment is under construction [37]. The combination of complementary detector systems, an air-shower array of electromagnetic and muon detectors complemented by a water-Cherenkov detector array in the center, is expected to make this the most accurate air-shower array in the Northern hemisphere.

New space experiments for direct cosmic-ray detections will increase the overlap in energy range with indirect measurements. 
The CTA Observatory [38], with both a northern site in La Palma, Canary Islands, and a southern one in Chile, will provide higher quality data and more open access both to the data and to the analysis tools of Imaging Cherenkov telescopes.

Most of the upgrades and new experiments rely on hybrid measurements combining two or even more detection techniques. This trend to multi-hybrid detection is a rational choice, since higher statistics alone may be insufficient for further progress.

\section{Conclusions}

In the past few years significant progress has been made towards identifying and understanding cosmic particle accelerators. Nevertheless some critical, long-standing questions in the field remain unanswered, or only partially answered: what are the sources of UHECR, what is their mass composition, what mechanism accelerates $\mathrm{CR}$ beyond $\mathrm{PeV}$ energies, what can we infer about high-energy $\mathrm{CR}$ from secondary messengers like gamma rays and neutrinos.

Observations performed by current and planned experimental facilities, together with new analysis techniques and strongest collaboration between different experiments, have an opportunity to give definite answers to these questions. Yet, to fulfill this potential, it is necessary to undertake a number of essential steps towards experimental and theoretical progress.

A complete picture of the high-energy Universe needs to account for all messengers: the validity of high-energy cosmic ray models needs considering the full charged CR, photon, and neutrino data sets from as many experiments as possible.

\section{References}

[1] K. Greisen, Phys. Rev. Lett. 16 (1966) 748-750.

G. T. Zatsepin, V. A. Kuzmin, JETP Lett. 4 (1966) 78-80.

[2] A. De Angelis and M. Pimenta, Introduction to Particle and Astroparticle Physics, 2nd Ed., Springer.

[3] M. Aguilar et al. (AMS Collaboration), Phys. Rev. Lett. 122 (2019) 041102.

[4] M. Aguilar et al. (AMS Collaboration), Phys. Rev. Lett. 122 (2019) 101101.

[5] P. Lipari, arXiv:1902.06173.

[6] O. Adriani et al. (CALET Collaboration), Phys. Rev. Lett. 120 (2018) 261102.

[7] G. Ambrosi et al. (DAMPE Collaboration), Nature 552 (2017) 63.

[8] Y. Asaoka, Proceeding of Science (ICRC2019).

[9] Q. An et al. (DAMPE Collaboration), Science Advances 5 (2019) 9.

[10] A. Aab et al. (Pierre Auger Collaboration), Nucl. Instrum. Meth. A798 (2015) 172-213.

[11] P. Tinyakov (Telescope Array Collaboration) Nucl. Instrum. Meth. A742 (2014) 29-34.

[12] The Pierre Auger Collaboration, Science 357 (2017) 1266.

[13] K. Kawata et al. (Telescope Array Collaboration), EPJ Conf (UHECR 2018) 210 (2019) 01004.

[14] M. Tanabashi et al. (Particle Data Group), Phys. Rev. D 98 (2018) 030001. 
[15] A. Castellina, PoS (ICRC2019) 004.

[16] T. AbuZayyad et al., EPJ Conf (UHECR 2018) 210 (2019) 01002.

[17] F. G. Schroeder, PoS (ICRC2019) 030.

[18] H. Dembinski et al., PoS(ICRC2019) 235.

[19] W. B. Atwood et al. (Fermi-LAT Collaboration), Astrophys. J. 697 (2009) 1071.

[20] M. Tavani et al., Astron. Astrophys. 502 (2009) 995-1013.

[21] J. Chang et al., Astroparticle Physics 95 (2017) 6-24.

[22] A. U. Abeysekara et al., The Astrophysical Journal 843 (2017) 39.

[23] M. Amenomori et al., Astrophys. J. 525 (1999) L93.

[24] J. Aleksic et al. (MAGIC Collaboration), Astroparticle Physics 72 (2016) 61-75. J. Aleksic et al. (MAGIC Collaboration), Astroparticle Physics 72 (2016) 76-94.

[25] J. Holder et al. (VERITAS Collaboration) Astropart. Phys. 25 (2006) 391-401.

[26] F. Aharonian et al., A\&A 457 (2006) 899.

H. Prokoph (H.E.S.S. Collaboration) PoS(ICRC2019) 656.

[27] H.E.S.S. Collaboration, A\&A 612 (2018) A1-A3.

[28] H.E.S.S. Collaboration, Nature 531 (2016) 476-479.

[29] C. Fruck et al., PoS(ICRC2019) 680.

[30] A. U. Abeysekara et al., The Astrophysical Journal 881 (2019) 2.

[31] M. Amenomori et al. (Tibet Collaboration) Phys. Rev. Lett. 123 (2019) 051101.

[32] M. Peresano et al., PoS(ICRC2019) 759.

[33] IceCube, Fermi-LAT, MAGIC Collaborations et al., Science 361 (2018) eaat1378.

[34] E. Bernardini, these Proceedings.

[35] S. Ogio (for the Telescope Array collaboration) PoS(ICRC2019) 013.

[36] arXiv:1907.07737.

[37] H. He (for the LHAASO collaboration) PoS(ICRC2019) 693.

[38] https://www.cta-observatory.org/. 